\title{
Nitrofurantoin induced interstitial lung disease mimicking COVID-19 pneumonia: Case report and review of the literature
}

\author{
Nur Aleyna YETKIN (ID) \\ Neslihan CERRAH \\ $\operatorname{DEMIR}(I D)$
}

Clinic of Chest Diseases, Kayseri City Hospital, Kayseri, Turkey

Kayseri Şehir Hastanesi, Göğüs Hastalıkları Kliniği, Kayseri, Türkiye
Cite this article as: Yetkin NA, Cerrah Demir N. Nitrofurantoin induced interstitial lung disease mimicking COVID-19 pneumonia: Case report and review of the literature. Tuberk Toraks 2021;69(1):121-124.

\section{Yazışma Adresi (Address for Correspondence)}

\section{Dr. Nur Aleyna YETKIN}

Kayseri Şehir Hastanesi,

Göğüs Hastalıkları Kliniği,

KAYSERI - TÜRKIYE

e-mail: alleynakemik@gmail.com

(C) Copyright 2021 by Tuberculosis and Thorax.

Available on-line at www.tuberktoraks.org.com
To the Editor,

Interstitial lung disease is a group of diseases that affects the interstitium of the lungs and can lead to progressive fibrosis of the lungs. The severity of these pulmonary adverse effects may range from trivial hypersensitivity reactions to extensive and irreversible lung fibrosis leading to respiratory failure and death. The potential causes of interstitial lung disease are broad and include infection, malignancy, autoimmune/connective tissue diseases, inhaled substances, radiotherapy, and certain medications (1). Nitrofurantoin is one of the drugs that have been shown to cause interstitial lung disease. Diagnosis of drug-induced interstitial lung diseases is made by excluding other causes and by drug use history. Therefore, a detailed disease history should be taken, complete blood count, collagen tissue markers, complete urinalysis tests should be performed, and rheumatology consultation should be performed if necessary. Bronchoalveolar lavage and sometimes lung biopsy is helpful in diagnosis in selected cases.

COVID-19 mainly affects the respiratory system and can lead to respiratory failure of different severity. Using a swab from the nasopharynx and the oropharynx is the primary diagnostic tool. In addition, the detection of nucleic acid can be easily affected by many factors, which include insufficient cellular materials and, clinically, the improper extraction of the nucleic acid materials. These factors consequently susceptible the rRT-PCR to false-negative results (2). Even if the rRT-PCR test is negative, the diagnosis 
of COVID-19 can be made with the findings of thoracic tomography in the presence of clinical findings or a history of contact. Therefore, even if the PCR is negative, in cases with clinical (fever, cough, contact), if thoracic computed tomography (CT) is compatible, treatment can be considered sufficient to start. In previous studies, it was reported that the CT can be more sensitive than the PCR (3). Although we focused more on COVID-19 disease during the pandemic period, we should not ignore other diseases that affect the lungs.

In this report, we present a patient who was diagnosed as COVID-19 with clinical and radiological findings in another center presented with respiratory distress 2 weeks after receiving COVID-19 treatment and finally diagnosed with nitrofurantoin-induced lung disease (NILD).

A 58-year-old male, never-smoker patient with a history of bladder cancer had admitted to another hospital with 3 days history of dyspnea. He had no history of travel or direct contact with an individual with COVID-19. On admission, the patient had 39 degrees of fever. C- reactive protein (CRP) was $30.7 \mathrm{mg} / \mathrm{L}$, white blood cell count (WBC) was $11100 / \mathrm{mm}^{3}$ and creatine was $1.48 \mathrm{mg} / \mathrm{dL}$ in laboratory examination. Peripheral patchy infiltrations, ground-glass areas were present on the patient's thorax CT taken at the time of admission and was interpreted as typical COVID-19 (Figure 1). The patient was recieved levofloxacin (500 mg/day) and hydroxychloroquine (400 mg twice daily loading, then $200 \mathrm{mg} /$ day) at the same hospital. Two consecutive COVID-19 rRT-PCR test results were found as negative. He had been receiving prophylactic (50 mg once daily) nitrofurantoin treatment for 8 months because of recurrent urinary tract infections. Existing nitrofurantoin treatment was continued with the recommendation of urology due to the positive leukocyturia and nitrite in full urinalysis. The patient's dyspnea and consolidation in the posteroanterior chest $\mathrm{X}$-ray was increased despite the treatment and had been given favipiravir.

Fourteen days after her discharge, the patient applied to our emergency serivce due to respiratory distress. Respiratory rate was $25 / \mathrm{min}$, oxygen saturation was $77 \%$ in the room air, blood pressure was $127 / 89$ $\mathrm{mmHg}$. Bilateral patchy widespread infiltrations were present on the PA chest X-ray at the time of presentation. The CT showed widespread patch-style ground-glass densities in both lungs, prominent peripheral honeycomb lung appearance in the upper lobes, and nodular areas with ground-glass density in the lung. Respect to the to the previous thorax CT findings, deterioration was observed (Figure 2). Firstly, considering the pre-diagnosis of COVID-19 induced fibrosis, the COVID-19 test was repeated and evaluated as negative. Spirometry and pulmonary function tests were not able to perform due to respiratory failure. Collagen tissue markers were found to be negative. In the repeated blood analysis, CRP was 12.7 $\mathrm{mg} / \mathrm{L}, \mathrm{WBC}$ was $8700 / \mathrm{mm}^{3}$ and creatine was 1.67 $\mathrm{mg} / \mathrm{dL}$. Nitrite and leukocyte esterase were negative in the full urine test.

The patient was admitted to the intensive care unit due to respiratory failure and underwent non-invasive mechanical ventilation. The patient did not have a history of occupational disease or exposure that could cause lung fibrosis. As a result, a diagnosis of drug-induced interstitial lung disease due to the empirical use of nitrofurantoin was made. Nitrofurantoin was immediately stopped. The patient was received methylprednisolone $100 \mathrm{mg} /$ day and broad-spectrum antibiotics was initiated based on the

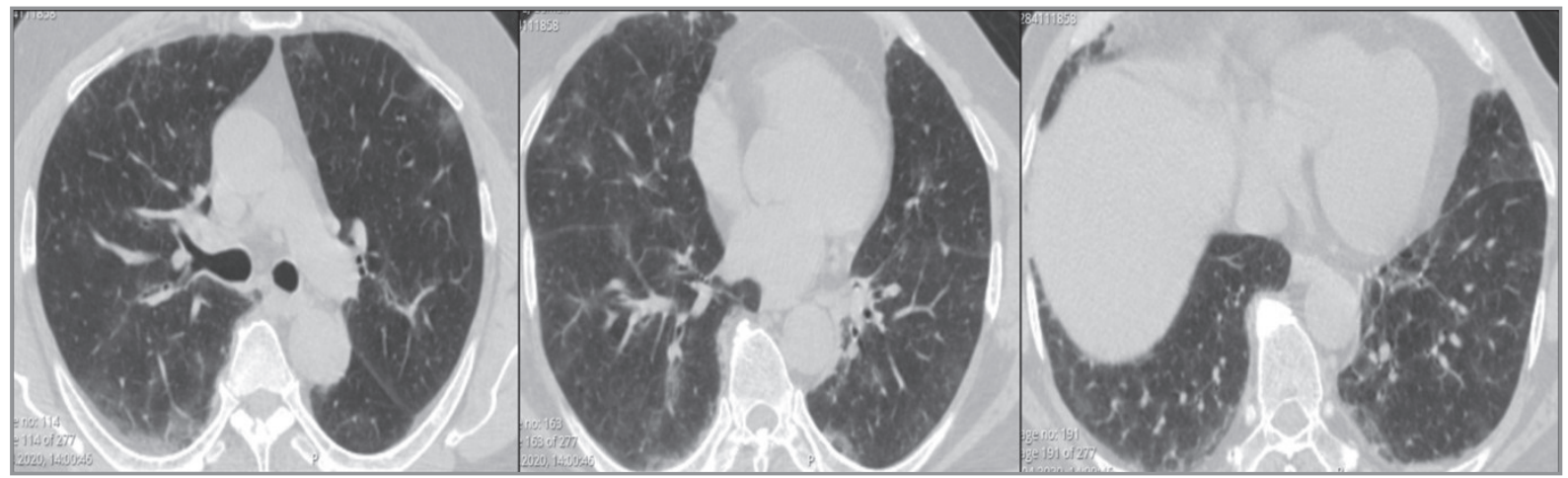

Figure 1. On the initial tomography, bilateral heterogeneous areas in common peripheral locally consolidated and mostly faint ground glass densities; interlobular septal thickening in both subpleural lung areas. 


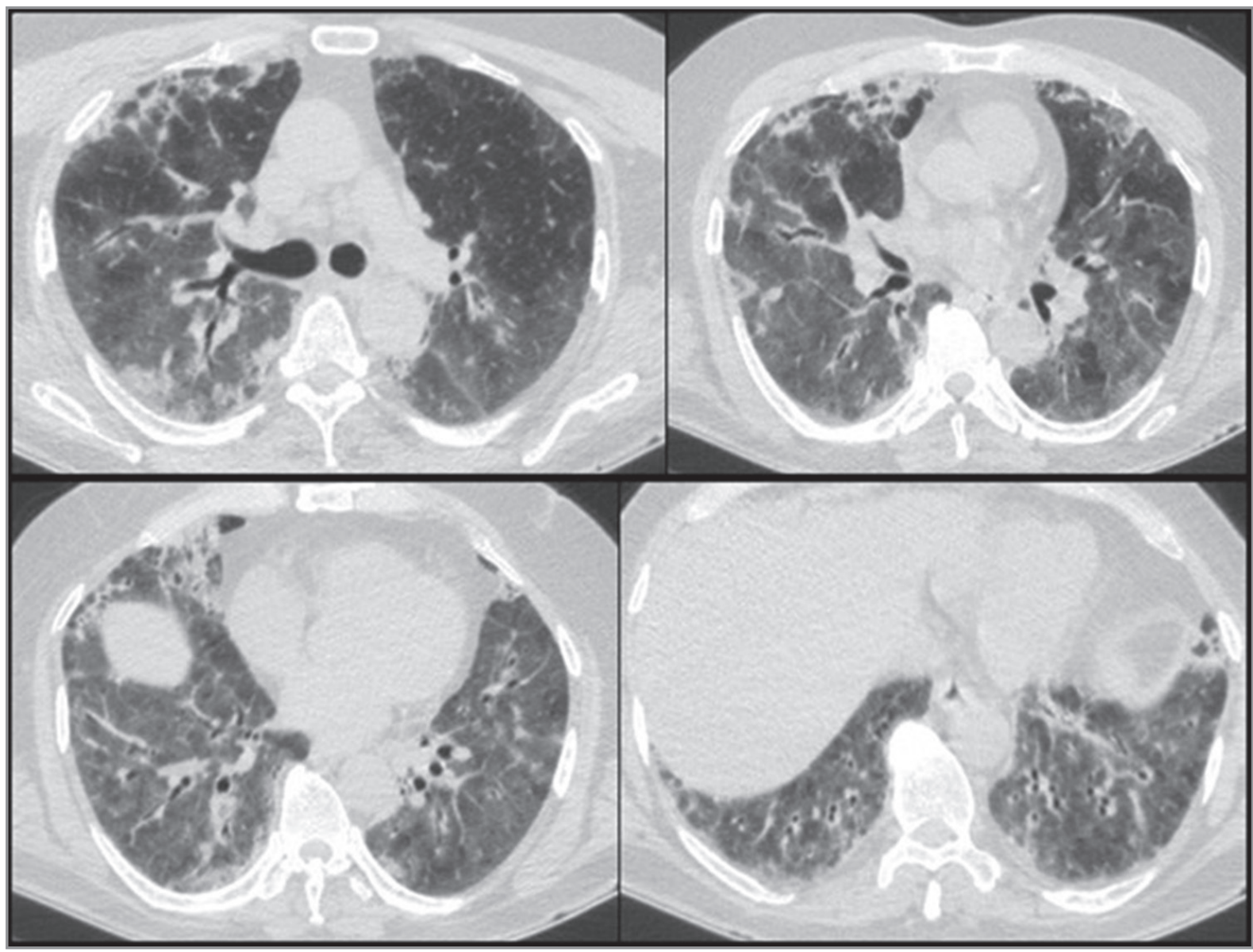

Figure 2. Subsequent CT revealed widespread patch-style ground-glass densities in both lungs, prominent peripheral honeycomb lung appearance in the upper lobes, and nodular areas with ground-glass density in both lungs.

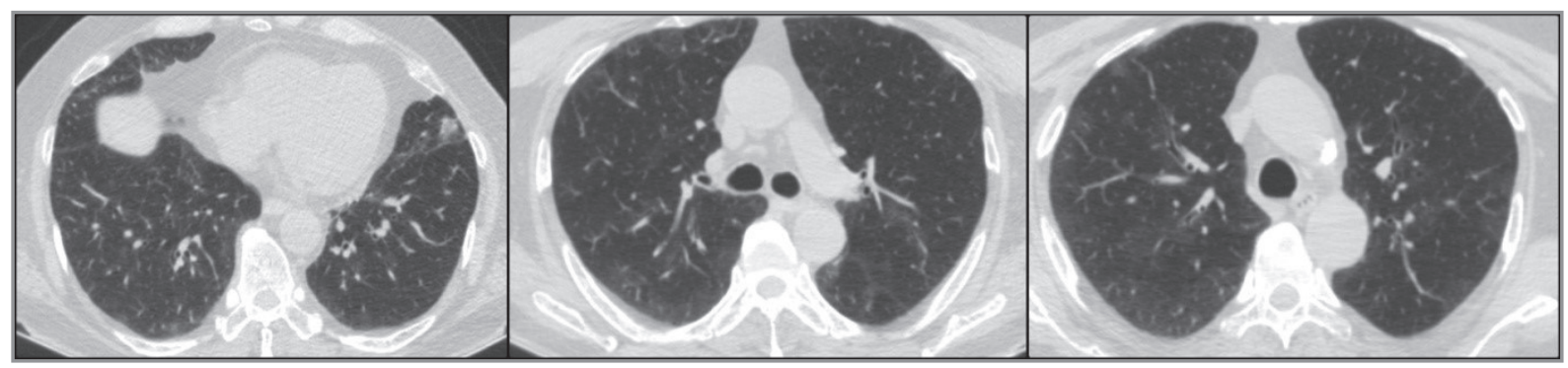

Figure 3. Control CT one month after steroid treatment.

high CRP levels. Patient's clinical findings were improved, and steroid dose was tapered. There was no need for NIMV first, so the oxygen supply was stopped. The patient was discharged with $32 \mathrm{mg} /$ day oral methylprednisolone. Radiological findings recovered almost completely on the CT scan acquired one month later (Figure 3).

Nitrofurantoin-induced lung disease (NILD) was first reported by Fisk A. et al. (4) in 1957 who described an acute self-limiting reaction to nitrofurantoin characterized by fever, dyspnea, cough, chest pain, rash, arthralgia, tachycardia, and tachypnea. Although the incidence of nitrofurantoin-related lung injury is estimated to be between 1 in 550 to 1 in 5400, it is probably underdiagnosed (5). Acute pulmonary damage that may develop even in small doses and usually it is not dose-dependent (6). The chronic form of pulmonary injury induced by nitrofurantoin is thought 
to occur due to a cell-mediated or toxic response to the drug and can result in NILD. Also, there have been previously reported cases of hepatotoxicity with lung fibrosis (7). The exact nitrofurantoin dose to cause chronic pulmonary injury was uncertain in a previously published 18 case study, but the amount of injury was dose dependent (8). The main treatment is to discontinue nitrofurantoin and it has been reported that steroids may be beneficial in cases with excessive fibrosis, like our case $(5,8)$. The diagnosis of drug-induced lung disease is usually based on circumstantial evidence, including the temporal relationship of drug administration to the onset of symptoms, improvement following the withdrawal of the drug, and knowledge of a particular drug's potential pulmonary toxicity. Granulomatous interstitial pneumonia has been reported in only one patient's biopsy in the literature (9). The histological features of drug-related lung disease were not specific and rarely diagnosed, so as in our case a lung biopsy was not performed in most patients with drug-related lung disease.

Since the patient was admitted in the COVID-19 pandemic period, despite the negative rRT-PCR results, he received COVID-19 treatment because of the fever and the radiological findings. Clinicians usually focus more on COVID-19 when questioning the patient, evaluating laboratory and radiological findings during the pandemic. It should not be forgotten that there are many infectious or noninfectious pulmonary diseases that cause imaging findings like COVID-19 pneumonia. As the pandemic extends, it seems that it will be more common to overlook dis- eases associated with COVID. It is important to carefully evaluate the patient's history, clinical and imaging findings to avoid misdiagnosis.

\section{REFERENCES}

1. Trusculescu AA, Manolescu D, Tudorache E, Oancea C. Deep learning in interstitial lung disease-how long until daily practice. Eur Radiol 2020; 30: 6285-92.

2. Chen D, Jiang X, Hong Y, Wen Z, Wei S, Peng G, et al. Can chest $C T$ features distinguish patients with negative from those with positive initial RT-PCR results for coronavirus disease (COVID-19)? AJR Am / Roentgenol 2020: 1-5.

3. Ai T, Yang Z, Hou $H$, Zhan $C$, Chen $C, L v W$, et al. Correlation of chest CT and RT-PCR testing in coronavirus disease 2019 (COVID-19) in China: a report of 1014 cases. Radiol 2020; 200642.

4. Fisk AA. Anaphylactoid reaction to nitrofurantoin. $N$ Engl J Med 1957; 256(22): 1054.

5. Santos Seoane SM, Fernández-Madera-Martínez $R$, Taboada-Martínez ML. Reversible pulmonary toxicity by nitrofurantoin. Rev Clin Esp 2020; 220(1): 69-70.

6. Mir E, Malik JA, Lone SA, Mohi-Ud-Din R, Khalil M. Spontaneous resolution of nitrofurantoin-induced chronic pulmonary toxicity presenting with respiratory failure. Adv Respir Med 2017; 85(6): 333-8.

7. Koulaouzidis A, Bhat S, Moschos J, Tan C, De Ramon A. Nitrofurantoin-induced lung-and hepatotoxicity. Ann Hepatol 2007; 6(2): 119-21.

8. Mendez IL, Nadrous HF, Hartman TE, Ryu JH. Chronic nitrofurantoin-induced lung disease. Mayo Clin Proc 2005; 80(10): 1298-302.

9. Sakata KK, Larsen BT, Boland JM, Palen B, Muhm Sr JR, Helmers RA, et al. Nitrofurantoin-induced granulomatous interstitial pneumonia. Int J Surg Pathol 2014; 22(4): 3527. 